\title{
Composite laminate under influence of temperature and moisture
}

\author{
Eva Kormaníková ${ }^{1, a}$ and Kamila Kotrasová ${ }^{1}$ \\ ${ }^{1}$ TUKE, Faculty of Civil Engineering, Department of Structural Mechanics, Vysokoškolská 4, 04200Košice, Slovakia
}

\begin{abstract}
The static response of simply supported carbon/epoxy composite plates in a hygrothermal environment is carried out using the general purpose finite element program COSMOS. The static load model and the hygrothermal incorporation are solved separately. The hygrothermal effect is introduced by using empirical relations for degrading the material stiffness properties of the matrix. The corresponding ply properties are calculated using Mori-Tanaka method. A parametric study is conducted by varying the fiber volume fraction and the fiber orientation of the angle plies in the laminate.
\end{abstract}

\section{Introduction}

The use of composite materials in the modern engineering applications has been increasing rapidly. Bridges, aerospace structures are few examples of their application. Steel bridges are replaced by composite materials due to their superior qualities like higher strength-to-weight ratio.

A laminate is a stack of lamina, with different fiber orientations, bonded together to attain desired properties. Laminates can be classified as symmetric, unsymmetric, balanced and unbalanced composites. In a symmetric laminate, the material and orientation of layers above the mid plane are identical to those below. In a symmetric laminate the bending-stretching coupling is absent. This is not true for unsymmetric laminates.

The analysis of composite structures is more complex and several theories have been proposed for the analysis of laminated composites. Composite laminates have larger planar dimensions, so they can be treated as plate elements. Therefore, plate theories can be applied in the analysis of laminated composites and it is very important to study the response of these materials to environmental conditions like temperature and moisture.

As alternative to the experimental determination of material properties of fiber matrix composite material is usage of various homogenization techniques. Many analytical homogenization techniques are based on the equivalent eingenstrain method, which considers the problem of a single inclusion embedded in an infinite elastic medium. Homogenization has been accomplished by using various techniques including the Fourier series technique, variational principles etc. Most fiber matrix composites have random arrangement of the fibers [2] (Fig. 1).

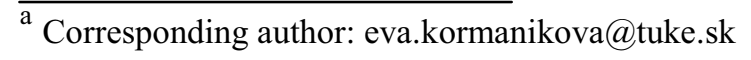

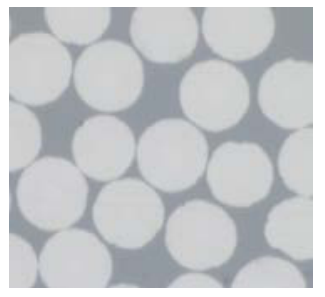

Figure 1. Randomly distributed fibers.

\section{Mori-Tanaka Method}

In the last decade, effective media theories, widely used in classical continuum micro mechanics, have been recognized as an attractive alternative to $\mathrm{FE}$ based methods. Since its introduction the Mori-Tanaka (MT) method has enjoyed a considerable interest in a variety of engineering applications. These include classical fiber matrix composites too [2].

General description of the Mori-Tanaka method in the framework of elasticity is treated in this section. The Mori-Tanaka method takes into account the effect of phase interactions on the local stresses by assuming an approximation in which the stress in each phase is equal to that of a single inclusion $r$ embedded into an unbounded matrix subjected to as yet unknown average matrix strain or stress.

The constitutive equation $\boldsymbol{\sigma}=\mathbf{C} \boldsymbol{\varepsilon}$ we can write in the following form:

$$
\left\{\begin{array}{l}
\sigma_{1} \\
\sigma_{2} \\
\sigma_{3} \\
\sigma_{4} \\
\sigma_{5} \\
\sigma_{6}
\end{array}\right\}=\left[\begin{array}{cccccc}
n & l & l & 0 & 0 & 0 \\
l & (k+m) & (k-m) & 0 & 0 & 0 \\
l & (k-m) & (k+m) & 0 & 0 & 0 \\
0 & 0 & 0 & m & 0 & 0 \\
0 & 0 & 0 & 0 & p & 0 \\
0 & 0 & 0 & 0 & 0 & p
\end{array}\right]\left\{\begin{array}{l}
\varepsilon_{1} \\
\varepsilon_{2} \\
\varepsilon_{3} \\
\varepsilon_{4} \\
\varepsilon_{5} \\
\varepsilon_{6}
\end{array}\right\}
$$


Material characteristics we can solve from the following equations:

$$
\begin{array}{r}
k=-\left(1 / G_{23}-4 / E_{22}+4 v_{12}^{2} / E_{11}\right)^{-1}, \quad l=2 k v_{12}, \\
m=G_{23}, \quad n=E_{11}+4 k v_{12}^{2}=E_{11}+l^{2} / k, \quad p=G_{12} .
\end{array}
$$

\section{Hygrothermal analysis}

Prolonged exposure to a hygrothermal environment has two major effects on a polymer matrix composite (PMC) structure. The first one is of a residual nature. The second significant effect of an increased hygrothermal condition is the degradation of the mechanical properties of a PMC ply. Again it is the matrix that bears the brunt of this degradation while the fiber properties remain nearly unchanged. The degradation occurs in both the stiffness and strength values of the matrix and consequently in the corresponding values of the lamina. In the present paper only this degradation effect is considered while ignoring the residual stresses. It is observed from various studies that the exposure to high moisture and temperature do not significantly affect the fiber related properties, but, the matrix properties get affected considerably. Therefore, the loss in performance can be related to temperature and moisture content of the matrix material.

In this paper, the relations given by Chamis $[1,3]$ are used to calculate the degraded properties of composite due to hygrothermal conditions. The hygrothermal strains in the longitudinal direction and transverse the fibre direction of a lamina are not equal since the effective elastic moduli $E_{L}$ and $E_{T}$ and also the thermal and moisture expansion coefficients $\boldsymbol{\alpha}, \boldsymbol{\beta}$ respectively, are different.

The stress-strain relations of a unidirectional (UD) lamina, including temperature differences are given [3]

$$
\left[\begin{array}{c}
\sigma_{x x}^{T} \\
\sigma_{y y}^{T} \\
\sigma_{x y}^{T}
\end{array}\right]=\left[\begin{array}{lll}
Q_{11} & Q_{12} & Q_{16} \\
Q_{21} & Q_{22} & Q_{26} \\
Q_{61} & Q_{62} & Q_{66}
\end{array}\right]\left[\begin{array}{c}
\varepsilon_{x x}^{0}+z \kappa_{x}-\alpha_{x} \Delta T \\
\varepsilon_{y y}^{0}+z \kappa_{y}-\alpha_{y} \Delta T \\
\gamma_{x y}^{0}+z \kappa_{x y}-\alpha_{x y} \Delta T
\end{array}\right]
$$

where $\Delta T$ is the temperature change, where $\bar{\varepsilon}$ and $\boldsymbol{\kappa}$ are vector of strain and curvature of mid-plane, respectively.

The internal forces in the laminate with the influence of temperature are following form

$$
\begin{gathered}
{\left[\begin{array}{l}
N_{x} \\
N_{y} \\
N_{x y}
\end{array}\right]=\left[\begin{array}{lll}
A_{11} & A_{12} & A_{16} \\
A_{21} & A_{22} & A_{26} \\
A_{61} & A_{62} & A_{66}
\end{array}\right]\left[\begin{array}{l}
\varepsilon_{x x}^{0} \\
\varepsilon_{y y}^{0} \\
\gamma_{x y}^{0}
\end{array}\right]+\left[\begin{array}{lll}
B_{11} & B_{12} & B_{16} \\
B_{21} & B_{22} & B_{26} \\
B_{61} & B_{62} & B_{66}
\end{array}\right]\left[\begin{array}{c}
\kappa_{x} \\
\kappa_{y} \\
\kappa_{x y}
\end{array}\right]-} \\
-\left[\begin{array}{lll}
A_{11} & A_{12} & A_{16} \\
A_{21} & A_{22} & A_{26} \\
A_{61} & A_{62} & A_{66}
\end{array}\right]\left[\begin{array}{l}
\alpha_{x} \\
\alpha_{y} \\
\alpha_{x y}
\end{array}\right] \Delta T=0 \\
{\left[\begin{array}{l}
M_{x} \\
M_{y} \\
M_{x y}
\end{array}\right]=\left[\begin{array}{lll}
B_{11} & B_{12} & B_{16} \\
B_{21} & B_{22} & B_{26} \\
B_{61} & B_{62} & B_{66}
\end{array}\right]\left[\begin{array}{l}
\varepsilon_{x x}^{0} \\
\varepsilon_{y y}^{0} \\
\gamma_{x y}^{0}
\end{array}\right]+\left[\begin{array}{lll}
D_{11} & D_{12} & D_{61} \\
D_{21} & D_{22} & D_{26} \\
D_{61} & D_{62} & D_{66}
\end{array}\right]\left[\begin{array}{l}
\kappa_{x} \\
\kappa_{y} \\
\kappa_{x y}
\end{array}\right]-}
\end{gathered}
$$

$$
-\left[\begin{array}{lll}
B_{11} & B_{12} & B_{16} \\
B_{21} & B_{22} & B_{26} \\
B_{61} & B_{62} & B_{66}
\end{array}\right]\left[\begin{array}{c}
\alpha_{x} \\
\alpha_{y} \\
\alpha_{x y}
\end{array}\right] \Delta T=0
$$

The stress-strain relations of a unidirectional (UD) lamina, including moisture differences are given

$$
\left[\begin{array}{c}
\sigma_{x x}^{H} \\
\sigma_{y y}^{H} \\
\sigma_{x y}^{H}
\end{array}\right]=\left[\begin{array}{lll}
Q_{11} & Q_{12} & Q_{16} \\
Q_{21} & Q_{22} & Q_{26} \\
Q_{61} & Q_{62} & Q_{66}
\end{array}\right]\left[\begin{array}{c}
\varepsilon_{x x}^{0}+z \kappa_{x}-\beta_{x} \Delta c \\
\varepsilon_{y y}^{0}+z \kappa_{y}-\beta_{y} \Delta c \\
\gamma_{x y}^{0}+z \kappa_{x y}-\beta_{x y} \Delta c
\end{array}\right]
$$

where $\Delta c$ is weight of moisture absorption per unit weight of the lamina.

The internal forces in the laminate with the influence of moisture are following form

$$
\begin{aligned}
& {\left[\begin{array}{l}
N_{x} \\
N_{y} \\
N_{x y}
\end{array}\right]=\left[\begin{array}{lll}
A_{11} & A_{12} & A_{16} \\
A_{21} & A_{22} & A_{26} \\
A_{61} & A_{62} & A_{66}
\end{array}\right]\left[\begin{array}{c}
\varepsilon_{x x}^{0} \\
\varepsilon_{y y}^{0} \\
\gamma_{x y}^{0}
\end{array}\right]+\left[\begin{array}{ccc}
B_{11} & B_{12} & B_{16} \\
B_{21} & B_{22} & B_{26} \\
B_{61} & B_{62} & B_{66}
\end{array}\right]\left[\begin{array}{c}
\kappa_{x} \\
\kappa_{y} \\
\kappa_{x y}
\end{array}\right]-} \\
& -\left[\begin{array}{ccc}
A_{11} & A_{12} & A_{16} \\
A_{21} & A_{22} & A_{26} \\
A_{61} & A_{62} & A_{66}
\end{array}\right]\left[\begin{array}{c}
\beta_{x} \\
\beta_{y} \\
\beta_{x y}
\end{array}\right] \Delta c=0 \\
& {\left[\begin{array}{l}
M_{x} \\
M_{y} \\
M_{x y}
\end{array}\right]=\left[\begin{array}{lll}
B_{11} & B_{12} & B_{16} \\
B_{21} & B_{22} & B_{26} \\
B_{61} & B_{62} & B_{66}
\end{array}\right]\left[\begin{array}{l}
\varepsilon_{x x}^{0} \\
\varepsilon_{y y}^{0} \\
\gamma_{x y}^{0}
\end{array}\right]+\left[\begin{array}{ccc}
D_{11} & D_{12} & D_{61} \\
D_{21} & D_{22} & D_{26} \\
D_{61} & D_{62} & D_{66}
\end{array}\right]\left[\begin{array}{c}
\kappa_{x} \\
\kappa_{y} \\
\kappa_{x y}
\end{array}\right]-} \\
& -\left[\begin{array}{lll}
B_{11} & B_{12} & B_{16} \\
B_{21} & B_{22} & B_{26} \\
B_{61} & B_{62} & B_{66}
\end{array}\right]\left[\begin{array}{l}
\beta_{x} \\
\beta_{y} \\
\beta_{x y}
\end{array}\right] \Delta c=0
\end{aligned}
$$

\section{Tsai-Wu Failure Criterion}

A more general form of the failure criterion for orthotropic materials under plane stress is expressed as

$$
F_{01} \sigma_{1}+F_{11} \sigma_{1}^{2}+2 F_{12} \sigma_{1} \sigma_{2}+F_{02} \sigma_{2}+F_{22} \sigma_{2}^{2}+F_{44} \tau_{12}^{2}<1
$$

where

$$
\begin{aligned}
& F_{01}=\frac{1}{X_{t}}-\frac{1}{X_{c}}, \quad F_{11}=\frac{1}{X_{t} X_{c}}, \quad F_{02}=\frac{1}{Y_{t}}-\frac{1}{Y_{c}}, \\
& F_{22}=\frac{1}{Y_{t} Y_{c}}, \quad F_{12}=-\frac{1}{2} \frac{1}{\sqrt{X_{t} X_{c} Y_{t} Y_{c}}}, \quad F_{44}=\frac{1}{S^{2}}
\end{aligned}
$$

The failure criterion for an orthotropic material under a strain is expressed as

$$
G_{01} \varepsilon_{1}+G_{11} \varepsilon_{1}^{2}+G_{12} \varepsilon_{1} \varepsilon_{2}+G_{02} \varepsilon_{2}+G_{22} \varepsilon_{2}^{2}+G_{44} \gamma_{12}^{2}<1
$$

where

$$
\begin{aligned}
& G_{01}=F_{01} E_{11}+F_{02} E_{12} \\
& G_{02}=F_{02} E_{22}+F_{01} E_{12}
\end{aligned}
$$




$$
\begin{gathered}
G_{11}=F_{11} E_{11}^{2}+F_{22} E_{12}^{2}+F_{12} E_{11} E_{12} \\
G_{22}=F_{22} E_{22}^{2}+F_{11} E_{12}^{2}+F_{12} E_{22} E_{12} \\
G_{12}=2 E_{12}\left(F_{11} E_{11}+F_{22} E_{22}\right)+2 F_{1}\left(E_{12}^{2}+E_{11} E_{22}\right) \\
G_{44}=F_{44} E_{44}^{2}
\end{gathered}
$$

When $F_{12}=\frac{-1}{2 X_{t}^{2}}$, the Tsai-Wu criterion is reduced to the Tsai-Hill criterion, and when $F_{12}=\frac{-1}{2 X_{t} X_{c}}$ the Tsai-Wu criterion is reduced to the Hoffman criterion [4, 7].

These failure criteria are used to calculate a failure index (FI) from the computed stresses and user-supplied material strengths. The failure index as a response of quantity is used for several FEA packages and it is defined as

$$
I_{F}=\frac{\text { stress }}{\text { strength }}
$$

Failure criteria predict the first occurrence of failure in one of the laminate layers. A value less than 1 denotes no failure and failure is predicted when IF $\geq 1$. The strength ratio is the inverse of the failure index.

It is important to distinguish between the fibre failure (FF) and the inter-fibre failure (IFF). In the case of shear plane stress, the IFF criteria discriminates three different modes [4]. The IFF Mode A is when perpendicular transversal cracks appear in the lamina under transverse tensile stress with or without in-plane shear stress. The IFF Mode B denotes perpendicular transversal cracks, but in this case they appear under in-plane shear stress with small transverse compression stress. The IFF Mode C indicates the start of oblique cracks when the material is under significant transversal compression.

The FF and the three IFF modes yield separate failure indices. The failure index for FF is defined as

$$
I_{F F}=\left\{\begin{array}{ccc}
\sigma_{1} / X_{t} & \text { if } & \sigma_{1}>0 \\
-\sigma_{1} / X_{c} & \text { if } & \sigma_{1}<0
\end{array}\right.
$$

For IFF with positive transverse stress, Mode A is active. The failure index in this case is defined as

$$
I_{I F F, A}=\sqrt{\left(\frac{\tau_{12}}{S}\right)^{2}+\left(1-p_{6 t} \frac{Y_{t}}{S}\right)^{2}\left(\frac{\sigma_{2}}{Y_{t}}\right)^{2}}+p_{6 t} \frac{\sigma_{2}}{S} \quad \text { if } \quad \sigma_{2} \geq 0
$$

where $p_{6 t}=0.3$.

Under negative transverse stress, either Mode B or Mode $\mathrm{C}$ is active, depending on the relationship between inplane shear stress and transversal shear stress. The failure indices are defined as

$$
I_{I F F, B}=\frac{1}{S}\left[\sqrt{\tau_{12}^{2}+\left(p_{6 c} \sigma_{2}\right)^{2}}+p_{6 c} \sigma_{2}\right] \quad \text { if }\left\{\begin{array}{c}
\sigma_{2}<0 \\
\left|\frac{\sigma_{2}}{\tau_{12}}\right| \leq \frac{F_{2 A}}{F_{6 A}}
\end{array}\right.
$$

$$
I_{I F F, C}=-\frac{Y_{c}}{\sigma_{2}}\left[\sqrt{\left(\frac{\tau_{12}}{2\left(1+p_{2 c}\right) S}\right)^{2}+\left(\frac{\sigma_{2}}{Y_{c}}\right)^{2}}\right] \text { if }\left\{\left|\frac{\sigma_{2}}{\tau_{12}}\right| \geq \frac{F_{2 A}}{F_{6 A}}\right.
$$

where $p_{6 c}=0.2$.

The limit between Mode B and Mode C is defined by the relation $F_{2 A} / F_{6 A}$,

where

$$
\begin{array}{r}
F_{2 A}=\frac{S}{2 p_{6 c}}\left[\sqrt{1+2 p_{6 c} \frac{Y_{c}}{S}}-1\right] \\
F_{6 A}=S \sqrt{1+2 p_{2 c}} \quad p_{2 c}=p_{6 c} \frac{F_{2 A}}{S} .
\end{array}
$$

\section{Numerical example and results}

It was considered the laminate made of 8 layers $[0 / \theta /-$ $\theta / 90]_{\mathrm{s}}$ and $[0 / \theta /-\theta / 90 / 0 / \theta /-\theta / 90 /]$. Constituent properties of Carbon fiber and Epoxy matrix are listed in the Table 1. Each Carbon/epoxy composite layer has the same material characteristics, that were obtained from Mori Tanaka method and listed in the Tables 2 and 3. The temperature change $\Delta T=20^{\circ} \mathrm{C}$ and moisture change $\Delta c$ $=1.5 \%$. The reinforcement angle $\theta$ is changed from $0^{\circ}$ to $90^{\circ}$. The results of hygrothermal conditions are solved for simply supported square laminate plate with $L=0.8 \mathrm{~m}$ and thickness $h=0.008 \mathrm{~m}$.

Table 1. Constituent mechanical properties of Carbon fiber and Epoxy matrix.

\begin{tabular}{|c|c|c|}
\hline Property & Carbon fiber & $\begin{array}{c}\text { Epoxy } \\
\text { matrix } \\
\text { (Dry) }\end{array}$ \\
\hline $\begin{array}{c}\text { Modulus of elasticity, } \\
E[\mathrm{GPa}]\end{array}$ & 228 & $3 \mathrm{GPa}$ \\
\hline Poisson's ratio, $v[-]$ & 0.3 & 0.36 \\
\hline
\end{tabular}

Table 2. Mechanical properties of Carbon/epoxy composite layer.

\begin{tabular}{|c|c|c|c|c|}
\hline $\begin{array}{c}E_{1} \\
{[\mathrm{MPa}]}\end{array}$ & $\begin{array}{c}E_{2} \\
{[\mathrm{MPa}]}\end{array}$ & $\begin{array}{c}G_{12} \\
{[\mathrm{MPa}]}\end{array}$ & $v_{12}[-]$ & $v_{21}[-]$ \\
\hline $1.38 \mathrm{e}+5$ & $1.032 \mathrm{e}+4$ & $4.389 \mathrm{e}+3$ & 0.343 & 0.0257 \\
\hline
\end{tabular}

Tables 3. Hygrothermal properties of Carbon/epoxy composite layer.

\begin{tabular}{|l|l|l|l|}
\hline $\begin{array}{l}\alpha_{1} \\
{\left[(\mathrm{~m} / \mathrm{m})^{\circ} \mathrm{C}\right]}\end{array}$ & $\alpha_{2}$ & $\beta_{1}$ & $\beta_{2}$ \\
{$\left[(\mathrm{~m} / \mathrm{m})^{\circ} \mathrm{C}\right]$} & {$[\mathrm{m} / \mathrm{m}]$} & {$[\mathrm{m} / \mathrm{m}]$} \\
\hline$-3.00 \mathrm{e}-7$ & $2.80 \mathrm{e}-5$ & 0.01 & 0.3 \\
\hline
\end{tabular}

\begin{tabular}{|l|l|l|l|l|}
\hline $\begin{array}{l}\sigma_{L u}^{t} \\
{[\mathrm{MP} a]}\end{array}$ & $\sigma_{L u}^{c}$ & $\sigma_{T u}^{t}$ & $\sigma_{T u}^{c}$ & $\sigma_{L T M}$ \\
{$[\mathrm{MP} a]$} & {$[\mathrm{MP} a]$} & {$[\mathrm{MP} a]$} & {$[\mathrm{MP} a]$} \\
\hline 2000 & 1200 & 50 & 170 & 70 \\
\hline
\end{tabular}




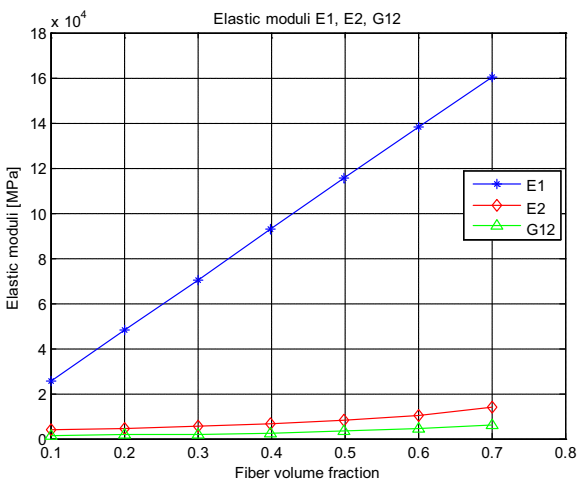

Figure 2. Elastic moduli versus fiber reinforced angle.

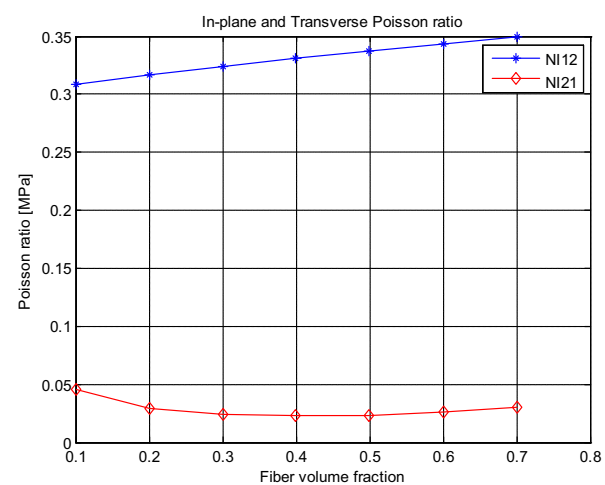

Figure 3. Poisson ratio versus fiber reinforced angle.

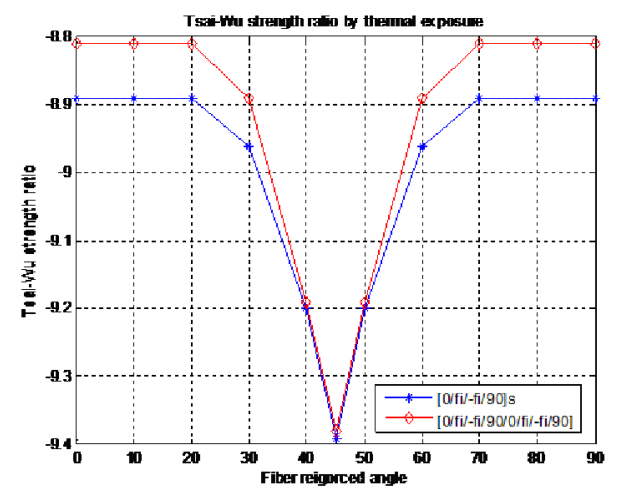

Figure 4. Moisture strength of ratio versus fiber reinforced angle.

Table 4. Bending moments of mechanical and hygrothermal exposure for $[0 / 45 /-45 / 90]_{\mathrm{s}}$.

\begin{tabular}{|c|c|c|c|}
\hline Property & $\begin{array}{c}M_{\mathrm{x}} \\
{[\mathrm{MNm} / \mathrm{m}]}\end{array}$ & $\begin{array}{c}M_{\mathrm{y}} \\
{[\mathrm{MNm} / \mathrm{m}]}\end{array}$ & $\begin{array}{c}M_{\mathrm{x}} \\
{[\mathrm{MNm} / \mathrm{m}]}\end{array}$ \\
\hline$q$ & $6.15 \cdot 10^{-5}$ & $2.33 \cdot 10^{-5}$ & $5.41 \cdot 10^{-5}$ \\
\hline$\Delta c$ & 0 & 0 & 0 \\
\hline$\Delta T$ & 0 & 0 & 0 \\
\hline
\end{tabular}

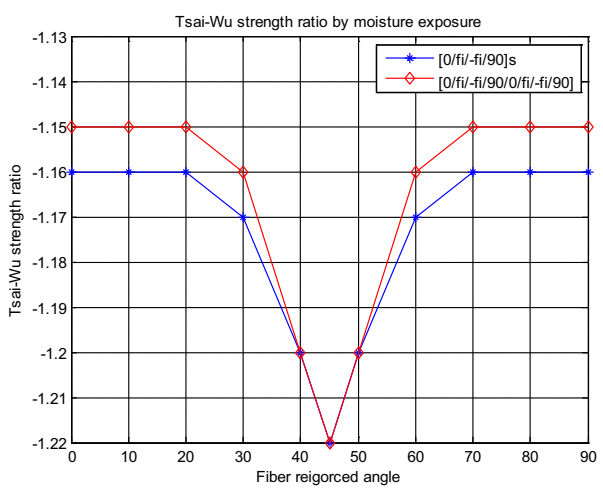

Figure 5. Thermal strength of ratio versus fiber reinforced angle.

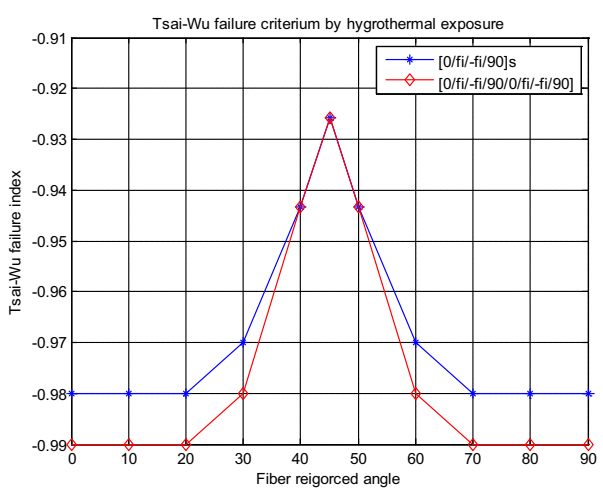

Figure 6. Mechanical and hygrothermal failure index versus fiber reinforced angle.

Table 5. Normal forces of mechanical and hygrothermal exposure for $[0 / 45 /-45 / 90]_{\mathrm{s}}$.

\begin{tabular}{|c|c|c|}
\hline Property & $\begin{array}{c}N_{\mathrm{x}}{ }^{=} N_{\mathrm{y}} \\
{\left[\mathrm{MN} / \mathrm{m}^{2}\right]}\end{array}$ & $\begin{array}{c}N_{\mathrm{xy}} \\
{\left[\mathrm{MN} / \mathrm{m}^{2}\right]}\end{array}$ \\
\hline$q$ & 0 & 0 \\
\hline$\Delta c$ & $3.37 \cdot 10^{-1}$ & 0 \\
\hline$\Delta T$ & $2.79 \cdot 10^{-2}$ & 0 \\
\hline
\end{tabular}

Table 6. Bending moments of mechanical and hygrothermal exposure for $[0 / 45 /-45 / 90 / 0 / 45 /-45 / 90 /]$.

\begin{tabular}{|c|c|c|c|}
\hline Property & $\begin{array}{c}M_{\mathrm{x}} \\
{[\mathrm{MNm} / \mathrm{m}]}\end{array}$ & $\begin{array}{c}M_{y} \\
{[\mathrm{MNm} / \mathrm{m}]}\end{array}$ & $\begin{array}{c}M_{x y} \\
{[\mathrm{MNm} / \mathrm{m}]}\end{array}$ \\
\hline$q$ & $1.2 \cdot 10^{-5}$ & $2.54 \cdot 10^{-5}$ & $-6.29 \cdot 10^{-6}$ \\
\hline$\Delta c$ & $3.1 \cdot 10^{-5}$ & $-3.1 \cdot 10^{-5}$ & $1.1 \cdot 10^{-5}$ \\
\hline$\Delta T$ & $1.39 \cdot 10^{-5}$ & $-1.39 \cdot 10^{-5}$ & $0.461 \cdot 10^{-5}$ \\
\hline
\end{tabular}

Table 7. Normal forces of mechanical and hygrothermal exposure for $[0 / 45 /-45 / 90 / 0 / 45 /-45 / 90 /]$.

\begin{tabular}{|c|c|c|c|}
\hline $\begin{array}{c}\text { Propert } \\
\mathrm{y}\end{array}$ & $\begin{array}{c}N_{x} \\
{\left[\mathrm{MN} / \mathrm{m}^{2}\right]}\end{array}$ & $\begin{array}{c}N_{y} \\
{\left[\mathrm{MN} / \mathrm{m}^{2}\right]}\end{array}$ & $\begin{array}{c}N_{x y} \\
{\left[\mathrm{MN} / \mathrm{m}^{2}\right]}\end{array}$ \\
\hline$q$ & $2.84 \cdot 10^{-2}$ & $3.22 \cdot 10^{-2}$ & $1.26 \cdot 10^{-3}$ \\
\hline$\Delta c$ & $3.37 .10^{-1}$ & $3.37 .10^{-1}$ & 0 \\
\hline$\Delta T$ & $2.79 .10^{-2}$ & $2.79 .10^{-2}$ & 0 \\
\hline
\end{tabular}




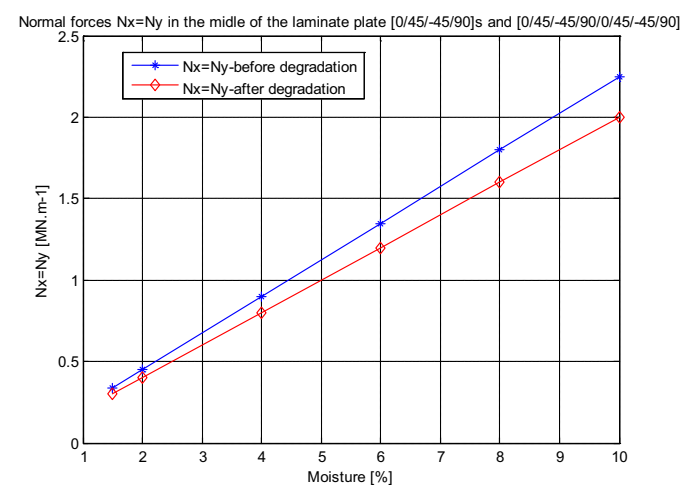

Figure 7. Normal forces $N_{x}=N_{y}$ of symmetric and asymmetric laminates versus change of moisture.

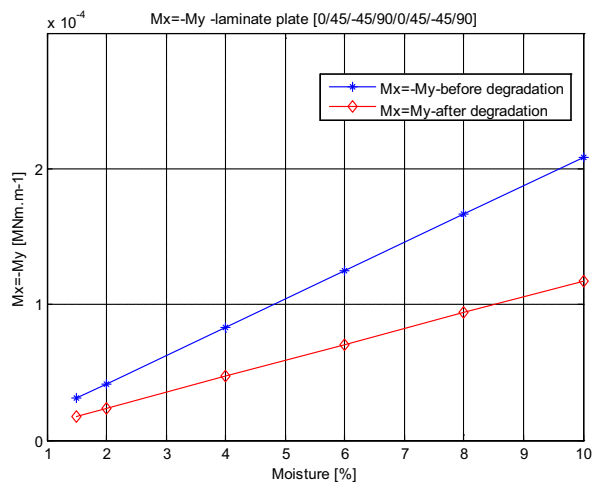

Figure 8. Bending moments $M_{x}=-M_{y}$ of unsymmetric laminates versus change of moisture.

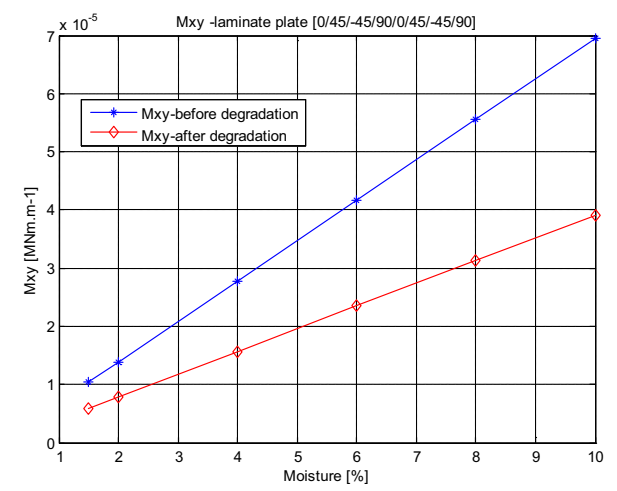

Figure 9. Bending moments $M_{x y}$ of unsymmetric laminates versus change of moisture.

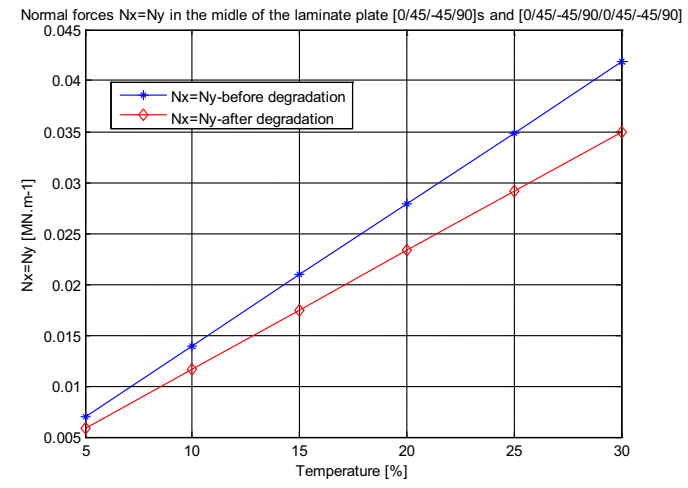

Figure 10. Normal forces $N_{x}=N_{y}$ of symmetric and asymmetric laminates versus change of temperature.

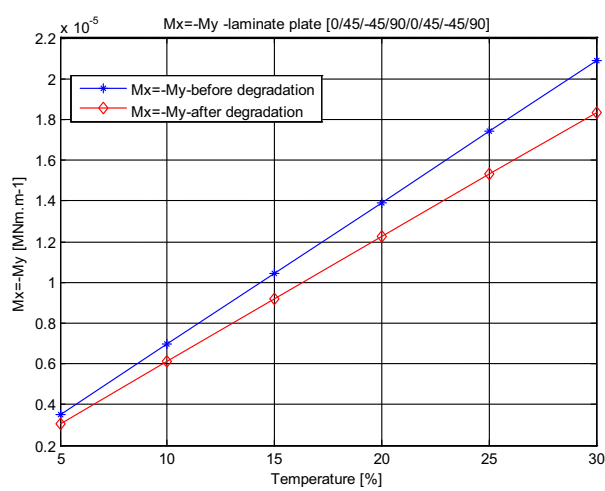

Figure 11. Bending moments $M_{x}=-M_{y}$ of unsymmetric laminates versus change of temperature.

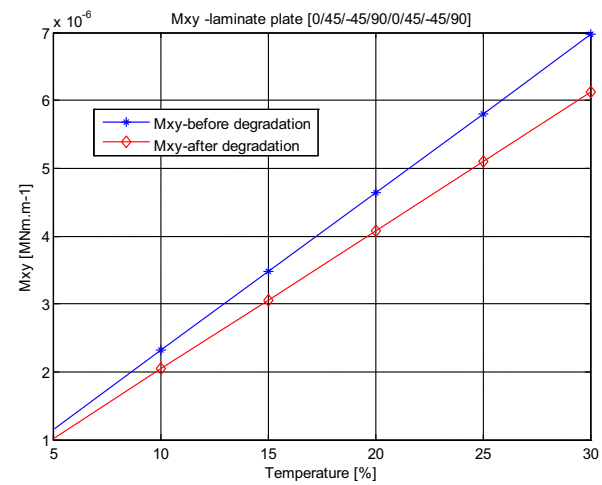

Figure 12. Bending moments $M_{x y}$ of asymmetric laminates versus change of temperature.

\section{Conclusion and discussion}

There was investigated the laminates made of 8 layers $[0 / \theta /-\theta / 90]_{\mathrm{s}}$ and $[0 / \theta /-\theta / 90 / 0 / \theta /-\theta / 90 /]$ in the paper. Each layer consists of Carbon fibers and Epoxy matrix. Effective material characteristics [11 - 13] for dry and wet laminate were establish using the program, that works under Mori-Tanaka method [2]. The material characteristics of undegraded laminate layer versus fiber volume fraction can be seen in Figs. 2, 3. The longitudinal modulus and longitudinal Poisson ratio have minor difference between undegraded and degraded 
values. The transverse modulus and in-plane shear modulus have considerable differences between undegraded and degraded values. For the optimization process $[5,6]$, the symmetric and unsymmetric laminates with fiber volume fraction 0.6 was taken into account. In the example there was calculated the influence of fiber reinforced angle change on strength ratio or failure index (Figs. 4-6). It is seen the best laminates are [0/45/-45/90] $\mathrm{S}$ and $[0 / 45 /-45 / 90 / 0 / 45 /-45 / 90 /]$.

The mechanical and hygrothermal analysis was performed using the program MATLAB. The response of laminated composite to hygrothermal conditions was investigated to validate the hygrothermal aspect of the model. The internal forces without degradation of material characteristics are written in the Tables $4-7$. The symmetric laminate has any coupling effect between normal forces and bending moments (Tabs. 4, 5), opposite the unsymmetric laminate with coupling effect (Tabs. 6, 7) [7 - 9]. Then the degradation process caused by temperature and moisture was investigated (Figs. 712). In the case of simply supported square laminate plates $[0 / 45 /-45 / 90]_{\mathrm{S}}$ and $[0 / 45 /-45 / 90 / 0 / 45 /-45 / 90]$ subjected to hygrothermal environment we can observe the difference between normal forces to $12.6 \%$ and between bending moment to $77.6 \%$. Based on these results a designer can choose the right ply orientations to control behavior of laminated plates [14 - 18]. It is possible to minimize the environmental effect by judiciously selecting the laminate configuration. Consequently, it is preferable to use the symmetric laminate panels for exterior utilization.

\section{Acknowledgment}

This work was supported by the Scientific Grant Agency of the Ministry of Education of Slovak Republic and the Slovak Academy of Sciences under Projects VEGA $1 / 0477 / 15$ and $1 / 0078 / 16$.

\section{References}

1. C. C. Chamis, Simplified Composite Micromechanics Equations for Hygral, Thermal and Mechanical Properties, Houston, TX, 1-10, (1983)

2. J. Sykora, M. Sejnoha, J. Sejnoha, Homogenization of coupled heat and moisture transport in masonry structures including interfaces, Appl. Math. and Comp. 219 (13), p. 7275-7285, (2013)

3. P. K. Kavipurapu, Forced Vibration and Hygrothermal Analysis of Composite Laminated Beams under the Action of Moving Loads, Morgantown, West Virginia, (2005)

4. V. Las, Mechanics of Composite Materials, West Bohemia University, (2008)

5. J. Kralik, Optimal design of npp containment protection against fuel container drop, Adv. Mat. Res. 688, p. 213-221, (2013)

6. M. Krejsa, P. Janas, I. Yilmaz, M. Marschalko, T. Bouchal, The use of the direct optimized probabilistic calculation method in design of bolt reinforcement for underground and mining workings,
The Scientific World Journal, Article number 267593, (2013)

7. M. Zmindak, Z. Pelagic, M. Bvoc, Analysis of high velocity impact on composite structures, Appl. Mech. and Mat. 617, p. 104-109, (2014)

8. K. Tvrda, Probability and sensitivity analysis of plate, Appl. Mech. and Mat. 617, p. 193-196, (2014)

9. J. Melcer, G. Lajcakova, Comparison of finite element and classical computing models of reinforcement pavement, Adv. Mat. Res. 969, p. 85$88,(2014)$

10. M. Sejnoha, J. Zeman, Micromechanical modeling of imperfect textile composites, Int. Jour. of Eng. Scien. 46 (6), p. 513-526, (2008)

11. J. Ma, S. Sahraee, P. Wriggers, , L. De Lorenzis, Stochastic multiscale homogenization analysis of heterogeneous materials under finite deformations with full uncertainty in the microstructure, Comp. Mech. 55, Issue 5, p. 819-835, (2015)

12. C. Maruccio, L. De Lorenzis, L. Persano, D. Pisignano, Computational homogenization of fibrous piezoelectric materials, Comp. Mech. 55, Issue 5, p. 983-998, (2015)

13. S. Tang, Y. Yang, X. H. Peng, X. X. Huang, K. Elkhodary, A semi-numerical algorithm for instability of compressible multilayered structures, Comp. Mech. 56, Issue 1, p. 63-75, (2015)

14. E. Kormanikova, I. Mamuzic, Optimization of laminates subjected to failure criterion, Metal. 50 (1), p. 41-44, (2011)

15. R. Dhabale, V. S. Jatti, Optimization of material removal rate of $\mathrm{AlMg} 1 \mathrm{SiCu}$ in turning operation using genetic algorithm, WSEAS Transactions on Appl. and Theor. Mech. 10, p. 95-101, (2015)

16. A. M. Valuev, Models and methods of multiobjective optimization in problems of quarry design and planning, WSEAS Trans. on Math., 13, p. 557-566, (2014)

17. E.A. Vorontsova, A projective separating plane method with additional clipping for non-smooth optimization, WSEAS Trans. on Math., 13, p. 115121, (2014)

18. Lovíšek, J. Králik, J., Optimal Control for ElastoOrthotropic Plate. Contr. and Cyber. 2, p. 219-278, (2006) 